\title{
Orbital Debris Ontology, Terminology, and Knowledge Modeling
}

\author{
Robert J. Rovetto $^{(1)}$, T.S. Kelso ${ }^{(2)}$, and Daniel A. O’Neil ${ }^{(3)}$ \\ ${ }^{(1)}$ Center for Orbital Debris Education and Research, University of Maryland, USA, rrovetto@terpalum.umd.edu \\ ${ }^{(1)}$ National Aeronautics and Space Administration (NASA) Datanauts open data initiative, USA \\ ${ }^{(2)}$ Center for Space Standards \& Innovation, ts.kelso@ centerforspace.com \\ ${ }^{(3)}$ NASA, daniel.a.oneil@nasa.gov
}

\begin{abstract}
The looming threat orbital debris poses to assets in orbit demands solutions. As the orbital population grows, so does this hazard, but so does the sea of data. The problem is also an opportunity for interdisciplinary innovation and cooperation. This paper focuses on the data and information management aspect of developing solutions for a sustainable and safe orbital space environment. The corresponding author's in-progress work to develop an orbital debris domain ontology is summarized in order to discuss knowledge modeling for this domain. Methodological approaches of this effort can also contribute to standards efforts and address terminological and policy questions.

Leveraging the growing volumes of orbital debris and space situational awareness (SSA) data will create a more complete picture of the orbital space environment. Part of the solution will be: consistent and correct data interpretation, sharing orbital debris and SSA data in one form or another, terminology development \& harmonization, and knowledge or domain modeling. To facilitate this, [Rovetto, 2015/16] discussed ontology development for the orbital debris domain. This paper lists concepts from that paper, and subsequently developed concepts [2-9].

Ontology engineering is an interdisciplinary field related to knowledge representation and reasoning in artificial intelligence, semantic technologies and the so-called semantic web. An ontology is effectively a computable and semantically rich terminology that presents a knowledge or domain model for a topic area. Expressions of knowledge or assertions are stored using formally defined term. This knowledge base is reasoned over to yield answers to queries, among other things. Ontologies have been developed in knowledge-based projects across various disciplines, and used for such things as search engines, chatbots, enterprise knowledge graphs, etc. Ontologies support: interoperability, automated reasoning, data sharing and integration, data search and retrieval, and communicating the meaning of data.
\end{abstract}

The Orbital Debris Ontology (ODO) [1], and related ontologies [Rovetto \& Kelso 2016] [Rovetto 2016, 2017], were proposed to help achieve this. ODO, for instance, is intended as a domain ontology that can be used across federated databases, offering an explicitly specified set of concepts describing the orbital debris domain. Its meaning-rich taxonomy will provide a sharable semantics for orbital debris data to, in part, consistently communicate the meaning of data to both humans and machines, and tag data elements in space object catalogs to help afford inference tasks, decision support, knowledge discovery, and information integration. ODO and the SSA ontology (SSAO) [2] is part of the overall Orbital Space Domain Ontology concept, which is conceived as a broader domain reference ontology. It aims to provide a knowledge representation structure of the orbital space environment, a common semantic model, and develop a sharable terminology. Collectively this will provide common meaning for datasets, a high-level taxonomy or classification for orbital space objects, and thus means to characterize space objects. Ongoing efforts have included using visualizations, R, JSON-LD, and contemporary semantic technologies.

Potential applications and interdisciplinary partnerships include web-based platforms, web apps, visualizations, and academia projects. Community input and participation may yield a more widely understood domain model as well as facilitate terminological standards. For example, the proposed conceptual, terminological and ontological analysis may contribute to such efforts as the Space Debris Mitigation Requirements in the International Standards Organization by developing more precise, consistent and coherent terms and definitions. Projects that seek to develop in-house ontologies can use ODO and related ontologies as domain reference ontologies.

This paper was developed independent of author affiliations. Readers are encouraged to contact corresponding author ${ }^{(1)}$ with general interest and potential opportunities to support or realize the described project. 


\section{INTRODUCTION}

The looming threat orbital debris poses to assets in orbit demands solutions. As the orbital population grows, so does this hazard, but so does the sea of data. This problem is also an opportunity for interdisciplinary innovation and cooperation. This paper focuses on the information management aspect of developing solutions for a sustainable and safe orbital space environment.

Leveraging the growing volumes of orbital debris and space situational awareness (SSA) data will create a more complete picture of the orbital space environment. Part of the solution will be: consistent and correct data interpretation, sharing orbital debris and SSA data, terminology harmonization, and knowledge or domain modeling. To facilitate this, [1] proposed ontology engineering for the orbital debris domain. This paper lists concepts from that paper, and subsequently developed concepts [2-9]. See https://purl.org/space-ontology.

The orbital debris, SSA and space traffic management (STM) communities, will benefit from formal analysis. Ontological (and philosophical) methods can help improve existing vocabularies, develop accurate classifications, fill in definitional gaps, corrects terminological inaccuracies, potentially address open policy questions, and improve standards efforts. Moreover, lack of shared vocabularies that are precise, coherent and consistent for not simply policy, but computational applications can be mitigated by adopting the proposed detailed, systematic approaches.

Terminologies are important for humans and machines. They promote communication and common understanding: meaning for humans, and formal syntax and semantics for machines. They support information access: tagging content and data elements for search and retrieval [12]. When transformed into taxonomies, greater organizational capability is acquired due to the structuring element. More powerful functionalities are achieved when taxonomies are given computable definitions, various interrelationships, logical axioms, and actionable rules. This advanced semantic structure is known as an ontology. With these added elements, it affords machine reasoning, data-sharing, data integration, semantic interoperability, etc. The Orbital Debris Ontology (ODO) [1] and related ontologies [2][5][6] ('ODO et al.' for short) are intended to facilitate these functions. Ontology engineering is interdisciplinary and related to artificial intelligence (AI), semantic technologies, linked data, and the semantic web. Ontologies have been developed in knowledge-based projects across various disciplines, and used for chatbots, search engines, enterprise knowledge graphs, etc. An ontology is both a computable semantically rich terminology, and a knowledge or domain model for a domain of interest. Knowledge is formalized and stored in processable expressions. The resulting knowledge base can be queried [13], potentially yielding new knowledge, quite significant for AI.

What, however, is orbital debris ontology, terminology, and knowledge modeling? First, orbital debris ontology [1][4] has various but related facets. It is the study of the generic aspects of orbital debris (from a philosophical perspective), and the development of computable ontologies and classifications to represent orbital debris (from a computational perspective). The orbital debris ontology is the computational ontology proposed in [1], whereas an orbital debris ontology is any computational ontology intended to represent orbital debris objects. Second, orbital debris terminology is more simply: terms used for orbital debris objects and related entities, e.g., those for categories of debris. Third, orbital debris knowledge modeling is the process of computationally modeling (or representing) orbital debris entities, data and knowledge thereof, which involves ontology development.

The ongoing work [1-9] summarized in this paper not only aims at the completion of a functional and accurate terminology for the community, but also classifications, and a dynamic ontological system that accurate represents the orbital space domain. By precisely formalizing the categories, interrelationships, and principles needed to describe the target domain at various levels of abstraction using sound and rigorous formal methods from philosophy, formal ontology and conceptual analysis, we will come to actionable human and machine readable models.

Section 2 describes the relationship between terminology, ontology and knowledge modeling. Section 3 discusses methodology. Section 4 summarizing ontology concepts of the Orbital Space Domain Ontology [5][6] to date. Section 5 mentions a successful application of ODO, offers ideas for applications (and partnerships), and discusses benefits of ontology. Finally, sections 6 and 7 list desiderata for this work and closing remarks, respectively. This paper was produced independent of the author affiliations.

\section{ONTOLOGY, TERMINOLOGY, AND KNOWLEDGE MODELING}

This section describes the interrelationship of ontology, terminologies, taxonomies, and knowledge modeling. Terminologies [12], taxonomies, and ontologies collectively help organize, analyze, find, describe, access and 
process an organizations content (e.g., enterprise data). Knowledge modeling, sometimes called 'semantic modeling', is about computationally representing knowledge. It encompasses various knowledge-based approaches, including semantic and AI technologies, which often utilize ontologies and taxonomies.

A central question in $\mathrm{AI}$ is how to represent knowledge in computer systems and artificial agents. This question is exemplified in knowledge representation and reasoning (KRR) [14], a subarea of AI and part of knowledge engineering, which includes ontology engineering. Ontologies provide models expressing knowledge so machines can better process and make sense of data [26], carryout tasks, and change behavior as needed. They are a semantically rich technology used with other systems to achieve goals within the knowledge management spectrum. Desired goals include automated reasoning (inference), classification, data search and retrieval, interoperability, data sharing and integration, knowledge discovery, and decision support. In recent years, ontologies or the aggregate of ontologies and other systems has been called a 'knowledge graph'. Since knowledge is revisable, KRR captures assertions, assumptions, beliefs, statements, intentions, etc. Implicit and explicit knowledge — both needed by AI systems - is formalized by an ontology. What more, both generic (universal) and particular (individual objects at specific times) knowledge are formalized. Examples include scientific principles of orbital dynamics, categorical knowledge about types of orbital debris; and ephemeris data about a tracked debris object, respectively. ODO and other ontologies mentioned in section 4 seek to capture this class-level and instance-level knowledge. To capture knowledge, both terms and definitions need to be formally specified in a computable language.

KRR use formal languages, sometimes called 'KR languages' or 'ontology languages', to capture knowledge. They have a precise syntax and formal semantics affording machine-readability. Natural expressions are translated into formal statements using the desired KR language. The ontology formally defines developer-specified terms (e.g. OrbitalDebrisObject) in the KR language. Ontologies thereby include a terminology, defining the categories, individuals, properties, and relations needed to describe a target domain. Inherent hierarchical constructs of an ontology give it a taxonomic form. Taxonomies themselves are terminologies hierarchically structured using a simple relation, e.g., broader-than, parent-child. Ontologies address limitations of taxonomies by including formal definitions, axioms, inference capability [15], a multitude of possible relationships, and thus greater semantic content. They give us the ability to conceptualize and computationally represent various interrelationships between domain objects (and the terms denoting them). Both hierarchical and non-hierarchical relations can be specified to thoroughly capture the domain. Ontologies, therefore, model domain entities and knowledge thereof, offering a formal representation of the domain. They, in part, enable an organization to more efficiently share data internally (as well as externally).

However, the expressivity of a KR language varies [28]: the more expressive the language, the more that can be said in it, and thus the closer the match to the intended idea or expression, i.e., the more accurate the model is. One form for representing knowledge is subject-predicate-object, mirroring basic English expressions, and used in semantic formalisms such as RDFS. Category (classes) and individual (instances) terms take the subject and object places, and terms expressing relationships the predicate place. This corresponds to noun and verb phrases. Each term has stable identifiers, affording reuse. A statement in this form is called a semantic triple, and a database of triples is called a triplestore. It is a simplified way to capture both generic and particular knowledge such as "Orbital debris fragment OD123 is located in low Earth orbit," "All resident space objects reside in an orbital regime," and "Low Earth Orbit is a type of orbital regime." An ontology formalizes such statements by using unary and n-ary constructs: classes and their instances are unary predicates, whereas relationships are binary or $n$-ary. Example formalizations include: has-Orbital-Regime(OD123, LowEarthOrbit), has-Orbital-Regime(RSO, OrbitalRegime), and is-a(LEO, OrbitalRegime). These constructs give an ontology the ability to model different interrelationships: those between a named individual and its characteristics, an individual and its class, between classes, etc. KRR often separately stores class-level, constraint, and relational information from instance-level statements about individuals, e.g., instance-of(OD123, OrbitalDebrisFragment). Collectively these databases have been called a knowledge base. Developers can assert actionable rules, sometimes based on first-order logic and in conditional (or if-then) statements to facilitate user-generated queries and automated inference capability.

Ephemeris data (instance data), can be stored in spreadsheets, and imported into an ontology that defines classes for the arguments of each formal statement, from which answers to specific data queries can be given. In other words, an organization's triplestore containing data on various aspects of orbital debris can be linked to an ontology such as ODO (section 4) to specify the meaning and constraints on the terms used for the triples (e.g. OrbitalInclination).

From semantically sparse terminologies to taxonomies and semantically rigorous ontologies, varying degrees of formality and semantics are possible for more advanced functionality. 


\section{METHODOLOGY SUMMARY}

Expressing intended concepts (meaning) is critical to developing an ontology. A term or label used for a concept is arguably secondary, but by no means trivial. It is needed for human readability and comprehension, so the developer should formulate terms that accurately match the intended meaning (and definition). The formal analysis proposed here will help do that by taking into account conceptual, philosophical, and ontological aspects. If nothing more, annotation properties in an ontology editor allow developers to specify alternative labels for a given concept.

Consider two examples to improve orbital debris terminology. First, the categories Space Debris and Orbital Debris are often equivalently used, but they are not identical. Orbital debris is a type of space debris from an ontological perspective. The former category contextually refers to debris in a specific environment or state of motion. From a terminological standpoint, there is no part of 'Space Debris' that singles-out debris in orbit, rather than debris elsewhere. Thus, it is more precise to use 'Space Debris' to signify debris in the outer space. Likewise, 'Orbital Space Debris' more accurately signifies debris in orbit. Second, although implicit usage of 'Orbital Debris' refers to Earth orbits, the term itself does not indicate that constraint in meaning. A qualifying element is needed, e.g., 'Earth Orbital Debris' as opposed to, say, 'Martian Orbital Debris'. This level of detail should afford ontologies such as ODO wider applicability. With further development, ODO et al. are intended to provide a higher-level classification system general enough for any celestial body or reference system. This does not exclude the possibility of an Earthspecific ODO, however. Simple improvements like these create a more accurate and computable classification. It supports computation because the ontology's taxonomy needs an accurate, logical structure to ensure efficient inheritance and inferences. Moreover, these corrections express truthful qualitative distinctions.

The present methodology, often used in ontology development, moves from domain knowledge research to concept and terminology development to ontology development, implementation, validation, and revision. The fact that natural language and our knowledge is fluid and revisable emphasizes the need for an iterative and revisable methodology (and ontology). An ontology should be consistent, coherent, with adequate coverage: it makes sense, is logical, represents intended meanings, and models a sufficient portion the target domain.

The first two stages involve understanding the chosen domain, and enumerating essential terms and domain concepts (the ideas we intend to express). Draw on intuitions about domain objects and ordinary words denoting them, while reviewing relevant literature and data resources to identify key domain terms and concepts. What concepts are expressed by that content? Review both generic (e.g., dictionaries) and discipline-specific resources (e.g., publications, textbooks, databases). Consult subject-matter experts, as well as ontologists (philosophical and computational). Terms include those for types of orbital debris (e.g. Fragmentation Debris) and for their various characteristics (or properties). Properties may be physical (e.g. mass), dynamic (e.g., attitude, (un)controlled motion, etc.) geometric, functional, social, legal, etc. Identify existing and formulate potential classifications for types of debris objects. Both qualitative and quantitative classifications for orbital debris should be developed. Properties are a typical criteria for characterization and class differentiation. Compare existing and potential definitions of the chosen terms. Define the chosen terms. Consulting multiple languages for variations in meaning may be insightful toward coming to a lingua franca for the domain. Term selection and definition can be supported by soliciting input from various orbital debris groups. For example, an open-source or community-driven approach, perhaps with an online platform with dedicated forums, has potential to improve the accuracy of the definitions, and provide for a more universally-acceptable vocabulary, classification, and knowledge model. The ontology development and implementation stages involves using ontology editor tools with a KR language to declare classes, relations, add definitions, constraints, axioms and rules. Individuals, e.g., various orbital debris objects in a given month, are added either manually or automatically by importing external documents housing instance data. Identify and select data sources to link to the ontology, e.g., orbital debris or space object catalogs. Verify and validate the ontology in part with competency questions (does it output desired information?), automated classification checks, and assessments of whether the intended goals and use-cases were achieved. Revise and iterate throughout.

The task of the ontologist is inherently interdisciplinary and abstract, making it suitable for philosophy backgrounds. They should take into account meaning, the real-world entities that terms actually or potentially refer to, the science of those entities, and their various facets from conceptual, linguistic, and ontological (analytical metaphysics) viewpoints. Some formal ontological facets include theories for generic and potentially invariant aspects of the world, such as causality, parthood, identity, and dependence. These can clarify aspects of domain-specific disciplines or theories. When such knowledge is computationally formalized they provide a shareable knowledge model usable across information systems, and that AI agents can draw on for behavioral and other actions.

The next section summarizes work for ODO et al. to date, including some features thereof. 


\section{THE ORBITAL SPACE DOMAIN ONTOLOGY: WORKS \& ONGOING EFFORTS}

The Orbital Debris Ontology (ODO) is part of a wider ontological goal of modeling the orbital space environment domain [5][6] which includes other overlapping SSA and STM entities (see https://purl.org/space-ontology). Table 1 lists published papers and efforts to date, including [1-11]. A deliverable of the overall project is the creation of both a domain reference ontology, and a suite of modular ontologies. A domain reference ontology provides various generic but domain-specific concepts and principles that may be invariant to subsets of the entities of that domain. These concepts can therefore subsume and annotate more specialized ones in other ontologies or information systems. The ontologies should be (re)usable by various actors as a backbone or core model for their content. Modular ontologies will cover other sub-domains such as orbital categories, orbitology, spacecraft and their parts, STM concepts, SSA and space domain awareness processes, etc.

Table 1. Summary of works to date.

\begin{tabular}{|c|c|}
\hline Paper or Presentation Year and Title & Summary \\
\hline $\begin{array}{l}\text { (2015) An Ontological Architecture for } \\
\text { Orbital Debris Data }\end{array}$ & $\begin{array}{l}\text { This paper discusses applying ontology to the orbital debris domain, } \\
\text { presenting the concept of an Orbital Debris Ontology (ODO) to model the } \\
\text { domain and facilitate data-sharing in the community. A preliminary } \\
\text { version of the ODO ontology file was introduced. [1] }\end{array}$ \\
\hline $\begin{array}{l}\text { (2016 with T.S. Kelso) Preliminaries of a } \\
\text { Space Situational Awareness Ontology }\end{array}$ & $\begin{array}{l}\text { This expands the domain of [1] by proposing a broader SSA domain } \\
\text { ontology (SSAO), which could have ODO (and other ontologies) as a } \\
\text { part. Preliminary version of the SSAO file was introduced. [2] }\end{array}$ \\
\hline $\begin{array}{l}\text { (2016) Ontology Architectures for the } \\
\text { Orbital Space Environment and Space } \\
\text { Situational Awareness Domain }\end{array}$ & $\begin{array}{l}\text { Various generic ontology architectures are presented. Each architecture is } \\
\text { visualized for the Orbital Debris and SSA domain, populating them with } \\
\text { ODO, the SSAO, and other concepts for ontologies (e.g., internal } \\
\text { ontologies, NASA- and ESA-specific ontologies, etc.). [3] }\end{array}$ \\
\hline $\begin{array}{l}\text { (2016) Orbital Space Environment and Space } \\
\text { Situational Awareness Domain Ontology }\end{array}$ & $\begin{array}{l}\text { A short paper and poster introducing the overall project and domain } \\
\text { reference ontology. [5] }\end{array}$ \\
\hline $\begin{array}{l}\text { (2016) The Orbital Space Environment and } \\
\text { Space Situational Awareness Domain } \\
\text { Ontology-Toward an International } \\
\text { Information System for Space Data }\end{array}$ & $\begin{array}{l}\text { A paper and poster expressing limitations of orbital data, and describing } \\
\text { the project concept as part of an envisioned international information } \\
\text { system. The system would aim to safeguard the orbital space environment } \\
\text { by establishing a knowledge-based data-sharing framework and domain } \\
\text { model. The authors vision of such an inter-organizational ontology-based } \\
\text { system with an orbital space visualization element is discussed. [6] }\end{array}$ \\
\hline (2016) Orbital Debris Ontology & $\begin{array}{l}\text { A power point presentation given as a panelist at the Center for Orbital } \\
\text { Debris Education and Research Workshop at University of Maryland. [4] }\end{array}$ \\
\hline $\begin{array}{l}\text { (2017) Ontology for Europe's Space } \\
\text { Situational Awareness Program }\end{array}$ & $\begin{array}{l}\text { An ontological architecture concept for the European Space Agency SSA } \\
\text { program. It shows how the ESA can either use the SSAO or create an } \\
\text { internal ESA SSA Ontology incorporating ODO and the SSAO. [9] }\end{array}$ \\
\hline (2017) An Ontology for Satellite Databases & $\begin{array}{l}\text { The Union of Concerned Scientists Satellite Database was used to show } \\
\text { how to develop an ontology for a given space data repository, focusing on } \\
\text { orbital objects and their orbital parameters [7]. An ontology implemented } \\
\text { in the Web Ontology Language was created. }\end{array}$ \\
\hline $\begin{array}{l}\text { (2017) Ontology-based Knowledge } \\
\text { Management for Space Data }\end{array}$ & $\begin{array}{l}\text { The relationship of ontology to knowledge management is discussed. } \\
\text { Various astronautical and astronomical projects using ontology were } \\
\text { summarized, including from NASA and the ESA. [8] }\end{array}$ \\
\hline (2017) Ontology & A live webinar presented as part of the NASA Datanauts [29] 2017 class. \\
\hline $\begin{array}{l}\text { (2017) Ontologies - Creating Human- } \\
\text { centered Taxonomies to Annotate Data" in } \\
\text { Data Discovery magazine }\end{array}$ & $\begin{array}{l}\text { This short online article [10] about the SSA ontology [2] was via the } \\
\text { corresponding authors engagement in the NASA Datanauts } 2017 \text { class. }\end{array}$ \\
\hline $\begin{array}{l}\text { (2017) Ontology Engineering for Space } \\
\text { Systems, Missions, and NASA Data, NASA } \\
\text { Fellowship Activity 2018, NNH18ZHA003N }\end{array}$ & $\begin{array}{l}\text { A submitted proposal for a NASA educational grant, customizing this } \\
\text { project for NASA data and space systems. ODO was included as an } \\
\text { ontology to be further developed and ideally used by NASA. [11] }\end{array}$ \\
\hline (2018-19 with Dr. Joanne Luciano) Big Data & A whitepaper proposing the project, customized for the sustainability and \\
\hline
\end{tabular}


in Space: Improving Spaceflight Safety through Knowledge-based Approaches

resilience themes in the University of Virgin Islands call for proposals.

Forthcoming works include: A NASA technical document with Dr. Daniel A. O'Neil of NASA's Marshall Spaceflight Center. A journal paper on space object ontology and taxonomy development is expected (an early draft has been available online since 2016). Future work includes an ontology and corresponding paper of the Two-line Element set.

Features of ODO et al. include the following. They are descriptive of the (sub)domain, adopt the open-world assumption, are revisable, modular, and reusable. Implementing them in more than one KR language, such as Common Logic, OWL, as well as conceptual modeling languages such as Object Role Model (ORM) [23], should allow use by a wider audience.

ODO, specifically, will represent types of orbital debris and their properties, including for tracked individual debris objects. Well-known qualitative orbital debris categories include: Fragmentation Debris, Inactive Payloads, Missionrelated Debris, Nonfunctional Spacecraft, Rocket Bodies, etc. The intended meaning for each is formalized with a specific ontological category and formal definition. Developing sub-categories for each will be worth exploring: what sub-classifications of, say, Fragmentation Debris are viable? What is the utility of such classifications? Quantitative aspects should be analyzed and incorporated to develop specialized categories of orbital debris. The astrodynamics community can inform the process.

Other ontologies include the SSAO, a Celestial Orbital Ontology (for classes of orbits), and a potential Space Traffic Management Ontology, as mentioned in [1-11]. The tentative architecture is that of a master ontology file composed of modular ontologies for specific sub-domains to capture the target domain of the orbital space environment (with acronyms along the lines of OSEDO, OSDO, or OSO for short). As further conceptual analysis and research is conducted to more precisely delineate the domain, and clarify domain concepts, the names of these ontologies may change to better reflect the sub-domain they are to model. For example, 'Space Situational Awareness Ontology', 'Space Domain Awareness Ontology', 'Space Domain Ontology', 'STM Ontology', etc. Any ontology files that have been created are subject to revision and not complete.

A module to annotate orbital data, e.g., ephemerides, TLEs, etc., will define the concepts shared among, say, dataproviders: orbital regime, orbital inclination, altitude, etc. As such it forms the basis for a shared knowledge model. If successfully usable by any orbital data source, data-providers can potentially interoperate with others.

Inference rules and axioms will help answer database queries and classify orbital debris into specific categories. For example, rules expressing invariant domain truths such as 'If an orbital debris object has an inclination of 90 degrees, then it is in a polar orbit.' are formalized. Rule and database query languages such as SWRL and SQRL will be employed. New information can be inferred given the formal interrelationships and classification schemes, making knowledge discovery a potential benefit. Patterns in behavior of orbital objects may be gleaned and categorized.

Spreadsheet (and other) documents serve more than one function. First, as data dictionaries (i.e., a term list with definitions and pertinent details). Second, to compare existing senses of domain terms such as 'orbital debris', 'spacecraft', 'space object', 'space situational awareness', 'orbit', etc. Third, to serve as a store for instance data to be imported into ontology editor tools such as Protégé (e.g., via its Celfie plugin).

Potential for community input is worth exploring to ensure accuracy of terms, definitions and the knowledge model expressed by these ontologies. The next section lists benefits of ontology and application ideas for ODO et al.

\section{BENEFITS AND POTENTIAL APPLICATIONS}

This section describes some benefits of ontology, and envisions collaborative applications for ODO et al. Potential applications and partnerships (5.1-5.8 below) include: web-based platforms, visualizations, academia projects, and standards and policy development.

A system with an ontological component can, in certain respects, be more easily modified. Ontology files are separate from system code, so changes to the ontology will propagate more smoothly, not requiring reprogramming. For example, modifying a class in an ontology is simpler than changing lines of code or even a relational database. Similarly, inference rules do not need to be in system code, but can be declared in an ontological platform. Finally, as domain knowledge changes, a properly developed ontology can be modified to reflect those changes. 
Ontologies can help make data FAIR [30] compliant, i.e., compliant with the FAIR data management principles. Ontologies provide the metadata and a semantic model to describe datasets. In combination with the KR language, they supporting the Interoperability principle. The globally unique identifiers being provided to ODO terms support the Findable principle. Together with the generic semantic model it supports reuse.

Machine learning (ML) has gained attention recently. Supplemented with ontologies, such as ODO et al., ML algorithms can improve their performance because ontologies provide already defined concepts and rules for algorithms to draw upon. It does not need to learn specific facts or relationships (e.g., that Fragmentation Debris is a kind of Orbital Debris), but only draw upon the ontology that has explicitly specified that background knowledge. ML needs data and well-tagged content. Ontology-annotated data may help glean insights into patterns within the data, while communicating what the data is about.

Inference capability is a benefit. The vocabulary or taxonomy defined in an ontology is used in user-generated queries (in a given query language, e.g., SPARQL) that an inference engine (or reasoner) processes to draw conclusions on data annotated with the categorical constructs (classes) of the ontology. Competency questions testing correctness are translated to formal queries. Possible queries are:

- Which debris objects have an altitude of X?

- What type of orbit has the most mission-related debris?

- How many collisions took place in LEO during time period T?
- Is orbital debris object 009 within LEO?

- Is there risk of collision between 009 and 010 ?

Ontological applications thereby provide decision-support capability, as well as information search and retrieval. Advanced reasoning for decision support may involve queries for conjunction assessment and warnings. One goal of the ODO et al. is to provide inference capability for orbital space domain awareness.

Linking, connecting, or bridging is a benefit of ontologies that can be expressed in various ways. For example, the vision of the semantic web is, in part, to connect various resources (documents, datasets, etc.). Ontologies are one tool to help do that. Debris data housed in spreadsheets, PDF files, etc., can be linked via the semantic tags provided by ontologies. Just as a taxonomy helps structure the unstructured content of an enterprise via controlled vocabularies and tagging, so do ontologies but with greater precision, functionality, and semantics. Unstructured isolated content, like documents of various formats, can be annotated with ontology classes to link resources for advanced information access.

Ontologies aim to solve a related problem: disparate databases and information systems of a common subject matter using different terms for the same meaning (or to refer to the same real-world object or data element). When these data silos are undesired, it can stifle communication (i.e., the knowledge level), interoperability (computer systems level), and data-sharing capability. It also inclines developers toward ad hoc or work-around solutions that are inefficient in the long run. Where distinct term are used to express the same meaning, an ontology can for example provide a broader class for each. This establishes a common link. Since they are external to the code, ontologies serve as a bridge - a common semantic language - between information systems, and other ontologies as well. The ability to interoperate with other ontologies is neither without challenges nor guarantee, but is helpful.

ODO et al. are intended to serve these roles, and will be developed to interoperate with other ontologies and data repositories alike. The common backbone knowledge model allows for consistent and common interpretation of data, because it allows user and machine to better understand the meaning of data. The shared representation helps link distinct systems. If accepted by a group as the standard semantic model or terminology, then that group has achieved a level of semantic interoperability. The ontology provides the agreed terms and definitions to describe the target domain, and affords computability.

Orbital data providers can therefore use ODO et al. to enrich their data (see 5.3, 5.4 below). The added semantic layer to orbital debris and SSA data unifies provider content under a knowledge model, which facilitates information integration, access and processing. Projects seeking to develop in-house ontologies can also use ODO in their knowledge graph or enterprise data architecture. The following sub-sections offers more concrete potential applications of ODO et al. We also mention a successful application of the SSAO (see 5.2) involving a 3D visualization.

\subsection{Ontology-based Orbital Debris Modeling Applications}

Although research is in order, we speculate that orbital debris modeling software, such as ORDEM [16], Debris Assessment Software [17] and LEGEND [18] from NASA's Orbital Debris Program Office, can benefit from ODO 
et al. Given that these ontologies are intended to provide a knowledge model of the target domain, the terms and concepts will overlap. Pertinent ontology classes may annotate data elements from each model.

\subsection{Web-based Applications and Visualizations}

A successful application of the SSAO is by Dr. Daniel A. O'Neil of NASA Marshall Spaceflight Center. He applied the SSAO to help generate a web-based interactive 3D visualization of the solar system [19], specifically utilizing orbit classes from the ontology. The development process included: using R to load JSON-LD and HTML libraries, importing orbital data from a spreadsheet file into an ontology editor to generate JSON-LD files for rendering a HTML table and the visualization. The complete process is being described in [27]. The vision for ODO et al. includes an ontology-based 3D visualization of the orbital space environment whereby classes annotate not only ephemerides and orbital space objects, but also the visual constructs in the graphical display.

\subsection{TLEs and CelesTrak - Orbital Data Formats}

The CelesTrak service [20], managed by Dr. T.S. Kelso, provides ephemeris data using the Two-line Element Set (TLE) format. CelesTrak can use ODO et al. to supplement that service, or develop a custom-designed ontology. By adding a downloadable ontology file to the website, users can annotate their data. For example, classes in an ontology of the TLE would annotate CelesTrak TLE data. It would also be usable by other TLE providers (and users). This idea is expressed in an in-progress paper by Rovetto.

\subsection{Space-Track}

Space-Track [21] can likewise potentially offer an ontology file for orbital data formats being used: TLE, State Vector, etc. The different file extensions (e.g. JSON) listed on their 'Examples' webpage relate to semantic technologies, and thus to ontology. Adding ontologies, such as ODO (or perhaps an ontology for the Conjunction Data Messages), in different file extensions, such as Common Logic, Web Ontology Languages (.owl extension), or the Object Role Model (.orm) [23], may benefit Space Track users.

\subsection{Video Games toward an Orbital Debris Removal Industry}

NASA's 2019 International Space Apps Challenge [22] included a challenge, managed by Dr. Daniel A. O'Neil, to develop an orbital debris mitigation video game. Resources used by teams included CelesTrak. Populated with individual entity data, and formatted in a standardized linked data format, such as JSON-LD, ODO could support the development of serious games. Conceptual designs and operational concepts could be evaluated and compared in video games. Additionally, video games could provide a non-government revenue stream for the development of an orbital debris removal industry. Further, ODO populated with TLE data could be exported to JSON-LD, convert to JSON, and used by JavaScript code in a video game that uses NASA's Web World Wind, or Analytical Graphic's Cesium platform.

\subsection{International Partnerships to Improve Orbital Space Environment Awareness}

Some recent industry and university projects from Asia to the Americas include ontological elements. They can use ODO et al. as a reference ontology, and potentially extending them with their own ontologies. NASA has a history of various knowledge-based projects using ontologies [8], while [9] offered a SSA ontology concept for the European Space Agency (ESA), and the ESA recently held a space system ontology workshop that mentioned the European Cooperation for Space Standardization eGlossary [24]. This glossary can be both used to generate an ontology, and mapped to the terminologies of other organizations toward creating a unified framework expressing shared meaning across the international community. It is one resource to compare domain terms as well.

\subsection{Standards Development and Policy}

Some associations aim to develop standards for the community. For example, the International Standards Organization has the Space Debris Mitigation Requirements, and The American Institute for Aeronautics and Astronautics has committees developing guidelines and standards for lexicons and recent ontology concepts. The formal analysis proposed here can contribute to these standards efforts by helping develop accurate terms and definitions for use in policy, legal and computational communities. The conceptual and philosophical analysis will ensure clarity, coherence, and consistency. Collaboratively developing these terminologies with those of the 
computational ontologies, e.g. ODO, may result in community consensus which should make both interoperability and the adoption of standards more likely.

\subsection{Avoiding Errors}

In a 2018 paper and presentation by T.S. Kelso and D. Oltrogge [25], they describe a case study in which an error was "due to incorrect reference frame used in operator's JSpOC-formatted data (ITRF vs. J2000)". Investigation is in order, but it may be worth exploring whether ontology can be applied to help avoid such errors. Can using the combined functionality of automated reasoning and semantic tags to label reference frame data avoid such errors? As it stands, ontologies can help an organization or project find clerical errors, e.g., in data labelling.

\section{DESIDERATA}

To fully realize this work, current desiderata include: sponsorship, partners, community involvement, applications, and data sources. Given the scale and interdisciplinary nature of the work, a multidisciplinary team environment (i.e., STEAM) is ideal. Since [1][2], projects interested in applying ontology have appeared in the global community. This represents potential partnerships, particularly university projects. Specific desiderata include: helping develop their own ontologies, or further develop (or otherwise use) ODO et al. in such projects. Other ontologies for the outer space domain can extend or import ODO et al. More specialized debris classifications can do likewise. Orbital datasets are also sought not only for domain research (section 3) but also to apply the ontologies. This makes partnerships with data providers of interest. A collaborative approach whereby members of the astronautical community provide input toward a model that reflects shared knowledge and is usable by orbital debris, SSA, and STM communities may benefit the project. Thus, ideas for community involvement, and opportunities to potentially establish a corresponding platform are desired. Ideas for applications are therefore desired. The reader is encouraged to contact the corresponding author ${ }^{(1)}$ with potential opportunities.

\section{CLOSING}

This paper briefly discussed the significance of terminology, ontology, and knowledge modeling for the orbital debris domain. It summarized an ongoing work [1-11], specifically the Orbital Debris Ontology and the broader Orbital Space Domain Ontology concept [5][6], and offered ideas for applications and partnerships. It aims at developing a knowledge model, defined terminologies, and a computable classification system that the orbital debris and space situational awareness community can use. Terminologies support the tagging of enterprise data.

Taxonomies add classificatory capability with a structuring relation. Ontologies afford more advanced functionality by logically formalizing the meaning of data, representing the domain, and modeling various interrelationships. The choice between formal terminologies, taxonomies and ontologies will depend on a given project and trade-offs, but ontologies provide capabilities that the former two structures do not. Orbital debris information management can reap the benefits of knowledge-based and AI approaches by applying ontology engineering. The formal analysiswhich draws on sound principles and method from philosophical, ontological, and conceptual perspectives-can not only help create accurate and coherent classifications and formal semantic models, but can also contribute to policy development by improve existing vocabularies, correcting inaccuracies therein, and filling definitional gaps.

\section{REFERENCES.}

1. Rovetto, R.J. An Ontological Architecture for Orbital Debris Data, Earth Science Informatics, Springer Berlin Heidelberg, Vo1. 9, Issue 1, pp. 67-82, 2015, 2016.

2. Rovetto, R.J. and Kelso, T.S. Preliminaries of a Space Situational Awareness Ontology, AIAA/AAS Spaceflight Mechanics meeting, Advances in Astronautical Sciences Vol. 158, 4177-4192, 2016.

3. Rovetto, R.J. Ontology Architectures for the Orbital Space Environment and Space Situational Awareness Domain, In CEUR Workshop Proceeding, Vol. 1660, Joint Ontology Workshops - Workshop on Ontology Modularity, Contextuality, and Evolution, Annecy, France, 2016.

4. Rovetto, R.J. “Orbital Debris Ontology” (Power Point Presentation), Center for Orbital Debris Education and Research (CODER) Workshop, University of Maryland, Aerospace Engineering Department, 15-17 November, 2016. Available at https://www.researchgate.net/publication/311707634_Orbital_Debris_Ontology. 
5. Rovetto, R.J. Orbital Space Environment and Space Situational Awareness Domain Ontology, In CEUR Workshop Proceedings, Vol. 1660, Joint Ontology Workshops - Early Career Symposium, France, 2016. Available at http://ceur-ws.org/Vol-1660/ecs-paper1.pdf, accessed 15 October 2019.

6. Rovetto, R.J. The Orbital Space Environment and Space Situational Awareness Domain Ontology -Toward an International Information System for Space Data, Advanced Maui Optical and Space Surveillance Technologies Conference, Maui, Hawaii, 2016. http://www.amostech.com/TechnicalPapers/2016/Poster/Rovetto.pdf.

7. Rovetto, R.J. An ontology for satellite databases, Earth Science Informatics, Springer Berlin Heidelberg, Vol.10 Issue 4, pp.417-427, 2017.

8. Rovetto, R.J. Ontology-based Knowledge Management for Space Data, 68th International Astronautical Congress, Adelaide, Australia, 2017.

9. Rovetto, R.J. Ontology for Europe's Space Situational Awareness Program, 7th European Conference on Space Debris. April 2017. https://conference.sdo.esoc.esa.int/proceedings/sdc7/paper/1046/SDC7-paper1046.pdf.

10. Rovetto, R.J. "Ontologies - Creating Human-centered Taxonomies to Annotate Data," Data Discovery Summer 2017, ISSUU, SecondMuse. Available at https://issuu.com/secondmuse/docs/nasa-datanauts-2017.

11. Rovetto, R.J. “Ontology Engineering for Space Systems, Missions, and NASA Data,” NASA Fellowship Activity 2018, NNH18ZHA003N, 2018.

12. W3C, "Vocabularies," https://www.w3.org/standards/semanticweb/ontology, accessed 11 November 2019.

13. W3C, "Queries," Available at https://www.w3.org/standards/semanticweb/query, accessed 11 November 2019

14. Brachman, R.J. and Levesque, H.J. Knowledge Representation and Reasoning, Elsevier, Amsterdam, 2014.

15. W3C, "Inference," Available at https://www.w3.org/standards/semanticweb/inference, accessed 11 Nov 2019

16. National Aeronautics and Space Administration (NASA), "ORDEM," Available at https://orbitaldebris.jsc.nasa.gov/modeling/ordem-3.0.html, accessed 11 November 2019.

17. NASA, "Debris Assessment Software" Available at https://orbitaldebris.jsc.nasa.gov/mitigation/debrisassessment-software.html, accessed 22 November 2019.

18. NASA, “LEGEND,” https://orbitaldebris.jsc.nasa.gov/modeling/legend.html, accessed 22 November 2019.

19. O’Neil, D.A. GitHub repository, Available at https://github.com/daoneil/spacemission, accessed 17 Nov 2019.

20. CelesTrak. Available at https://www.celestrak.com/, accessed 15 October 2019.

21. Space-Track. Available at https://www.space-track.org, accessed 17 November 2019.

22. "Orbital Scrap Metal - The Video Game, International Space Apps Challenge," Available at https://2019.spaceappschallenge.org/challenges/stars/orbital-scrap-metal-the-video-game/details.

23. “Object Role Model,” Available at http://www.orm.net/, accessed 17 November 2019.

24. European Cooperation for Space Standardization, “eGlossary,” https://ecss.nl/glossary/, accessed 17 Nov 2019.

25. Kelso, T.S., Oltrogge, D. The Need for Comparative SSA, $69^{\text {th }}$ International Astronautical Congress, Germany, 2018, Available at https://www.celestrak.com/publications/IAC/2018/IAC-18.A6.7.8.pdf, accessed 15 Oct 2019

26. Knight, M. "Taxonomy vs Ontology: Machine Learning Breakthroughs", Available at https://www.dataversity.net/taxonomy-vs-ontology-machine-learning-breakthroughs/

27. O’Neil, Daniel, Rovetto, R.J. An Ontology-Oriented Orrery, NASA (Forthcoming, TBD. Title Subject to change)

28. Soylu, A., De Causmaecker, P., Preuveneers, D., Berbers, Y., Desmet, P. Formal modelling, knowledge representation and reasoning for design and development of user-centric pervasive software: a meta-review, International Journal of Metadata, Semantics and Ontologies, Vol. 6, No. 2, 2011.

29. NASA Datanauts. Available at https://open.nasa.gov/explore/datanauts/, accessed 22 Nov 2019.

30. Go-FAIR, "Fair Principles," Available at https://www.go-fair.org/fair-principles/, accessed 22 Nov 2019. 